\title{
L'étude des perceptions des populations et pratiques de développement
}

\section{Ludovic Kibora}

\section{(2) OpenEdition \\ Journals}

Édition électronique

URL : http://journals.openedition.org/apad/4117

DOI : 10.4000/apad.4117

ISSN : 1950-6929

Éditeur

LIT Verlag

Édition imprimée

Date de publication : 1 janvier 2012

Référence électronique

Ludovic Kibora, «L'étude des perceptions des populations et pratiques de développement », Bulletin de I'APAD [En ligne], 34-36 | 2012, mis en ligne le 19 juillet 2013, consulté le 08 septembre 2020. URL: http://journals.openedition.org/apad/4117 ; DOI : https://doi.org/10.4000/apad.4117

Ce document a été généré automatiquement le 8 septembre 2020

Bulletin de l'APAD 


\title{
L'étude des perceptions des populations et pratiques de développement
}

\author{
Ludovic Kibora
}

\section{Introduction}

1 Le Burkina Faso, pays enclavé situé dans une zone de transition entre la région soudano-guinéenne et le Sahel, est classé parmi les pays les plus pauvres du monde selon l'indice du développement humain durable du PNUD. Environ $80 \%$ de la population vit dans les zones rurales et se consacrent principalement à l'agriculture (INSD, 2008). Cependant la précarité des ressources naturelles, la prédominance de technologies arriérées, et les aléas climatiques expliquent en partie la vulnérabilité structurelle de cette agriculture.

2 En 2003, l'Enquête Burkinabè sur les Conditions de vie des Ménages établissait que $46,4 \%$ de la population vivait en dessous du seuil officiel de pauvreté monétaire (INSD, 2003). Les estimations en 2008 indiquent une incidence de 42,8\% ${ }^{1}$. C'est pourquoi suite au bilan désastreux des Programmes d'ajustements structurels (PAS) des années 90, l'Etat burkinabé a adopté le Cadre Stratégique de Lutte contre la Pauvreté (CSLP) en 2000. Ce document révisé en 2003 a constitué jusqu'ici le cadre de référence en matière de politique de développement au Burkina Faso. Actuellement, un nouveau programme est en cours d'élaboration sur la base des résultats de ce CSLP.

3 Nous pouvons définir la pauvreté comme un phénomène polysémique dont la manifestation la plus générale est le dénuement profond associé à un état de manque et de privation relatif à un ensemble de besoins fondamentaux : revenus, nutrition, alimentation, accès aux services sociaux de base, possession d'actifs etc. Dans de nombreuses langues burkinabè les signes de la pauvreté font référence à 
l'habillement, l'incapacité physique, l'absence d'activité, le manque de ressources etc.

4 Certaines recherches estiment que, la situation de pauvreté d'un ménage peut provenir soit d'une baisse temporaire de son niveau de vie, soit de la faiblesse du niveau de vie sur une longue période (Jalan et Ravallion, 2000). En plus des différents critères matériels couramment utilisés pour décrire et comprendre la pauvreté, celle-ci est fortement liée aux facteurs psychosociaux et culturels de la société.

5 Ces différents aspects qui nécessitent une intervention de l'anthropologie ont été faiblement analysés dans de nombreux pays, particulièrement au Burkina Faso. En effet, au Burkina Faso les divergences entre quantitativistes et qualitativistes en matière d'appréciation de la pauvreté, ne portent pas sur le concept lui-même mais plutôt sur la mesure. L'insuffisance de la prise en compte de l'aspect qualitatif caractérise les recherches sur la pauvreté.

6 Certains auteurs assignent la persistance des individus ou ménages dans la pauvreté à des prédispositions psychologiques (Corcoran et al. 1985) ou a une conjugaison de facteurs sociaux, culturels, économiques (Sawadogo, 1997). Bien que ces recherches ne soient pas purement anthropologiques, elles situent le fait que la pauvreté soit la résultante des interactions de plusieurs facteurs et font référence à des aspects de perceptions.

7 L'intervention de l'anthropologie doit permettre d'articuler les dimensions sociales, politiques et spatiales et d'inscrire la pauvreté dans le contexte global des problèmes de notre société. De ce fait l'approche participative adoptée par l'anthropologue doit consister à recueillir le point de vue des populations sur leur propre situation, leurs croyances, leurs connaissances des moyens utilisés par les différents acteurs, etc. A partir d'exemples de cas burkinabè, nous essayerons de montrer comment l'étude des perceptions et attentes des populations est importante dans le processus l'élaboration d'une stratégie nationale de lutte contre la pauvreté.

\section{L'absence des données qualitatives dans les approches de lutte contre la pauvreté}

8 L'échec de nombreux projets et programmes de développement, souvent initiés au profit des populations africaines, rendent plus qu'actuel la question du développement comme nous le mentionnions plus haut. Ce concept de développement, rappelons-le, a fait l'objet de réflexions aussi variées que contradictoires de la part de différents chercheurs en sciences sociales (Hauban, 1982 ; Fergusson, 1990 ; Hobart, 1993 ; Olivier de Sardan, 1995 ; Little et Painter, 1995 ; Crush, 1995). Nous estimons avec Crush: « One of the basic impulse of those who write on development is a desire to define, categorize and bring order to a heterogeneous and constantly multiplying field of meaning. »(Crush; 1995:2) ${ }^{2}$.

9 Au Burkina Faso, de nombreuses stratégies nationales de développement ont été mises en œuvre depuis l'indépendance politique du pays en 1960. La plus significative est la promotion de l'auto-développement initié par le pouvoir révolutionnaire à partir de 1983. Très volontariste, cette pratique de 
développement visait à amener les populations à compter surtout sur leurs propres forces. En 1991, des réformes engagées avec les institutions de Bretton Woods ont abouti à la mise en place des programmes d'ajustement structurel (PAS) qui visaient à établir l'équilibre des finances publiques et orienter l'économie vers une économie de marché. On assiste alors à la mise en œuvre de politiques de déréglementation des prix, de privatisation d'entreprises publiques, de révision du code de travail, etc. Les résultats n'ont pas produit les effets escomptés. Malgré les taux de croissances positifs brandit par le pouvoir politique, les réalités quotidiennes des populations sont décevantes et se présentent en terme de faiblesse de la couverture sanitaire, de difficulté d'accès à l'éducation, de production agricole structurellement déficitaire. Parallèlement le recours régulier à l'aide extérieure ne fait que croître, de même que la paupérisation des populations notamment celles rurales. Les politiques de développement, pour n'avoir pas su intégrer des aspects autres que ceux purement économiques, se sont soldées par des échecs prévisibles. En l'absence d'une politique véritable de développement l'État burkinabè s'est engagé dans le processus des Cadres Stratégiques de Lutte contre la Pauvreté (CSLP) préconisés par les mêmes institutions initiatrices des PAS. Le CSLP burkinabè a été adopté en 2000 et révisé en 2003. La revue à mi-parcours en novembre 2008, effectuée par les partenaires techniques et financiers du Burkina Faso a abouti à un constat plus que mitigé, malgré l'auto satisfecit souvent affiché par les pouvoirs publics. De nombreux projets et programmes sectoriels soutenus par les partenaires bilatéraux et multilatéraux ont été mis en œuvre avec la même philosophie quantitativiste. La situation actuelle se caractérise par une forte baisse au niveau de la production cotonnière la principale culture de rente du pays, baisse due à la conséquence d'une conjugaison de plusieurs facteurs, notamment les conditions climatiques défavorables, la baisse des prix aux producteurs, la hausse des prix des intrants, les retards de paiement des paysans, etc.

10 Quant à l'agriculture vivrière, basée essentiellement sur la production céréalière, les progrès saisonniers se font de façon irrégulière. La situation de disparités entre les différentes provinces du pays est telle que les provinces excédentaires ne parviennent pas à servir les localités déficitaires, ce qui n'est pas sans risque de tensions inflationnistes. Le secteur de l'élevage bien qu'enregistrant des progrès dans le domaine de la production animalière et laitière, reste confronté à des difficultés liées à l'insuffisance d'aliments de bétail (sous-produits agroindustriels) et leur coût élevé.

11 Comme on peut le constater, ces principaux secteurs qui occupent plus de la moitié de la population du pays et constituent les pourvoyeurs des ressources nationales sont en difficultés permanentes. Cependant, ils continuent d'être appréhendés par les mêmes approches quantitativistes. Le nouveau cadre de développement qui est en cours d'élaboration n'échappe pas à cette dynamique. Même si de plus les différentes études tiennent compte du caractère multidimensionnel et même subjectif de la pauvreté. Les méthodes utilisées pour aboutir à ces résultats procèdent par questionnaire où le qualitatif est tiré des données quantitatives.

$12 \mathrm{Au}$ Burkina Faso, les études sur la pauvreté ont surtout été réalisées après l'adoption du Programme d'Ajustement Structurel (PAS) en 1991. Les mesures du 
PAS et les controverses sur leurs impacts sociaux notamment sur le bien-être des populations ont alimenté la production de données et d'analyses sur la pauvreté. Les deux études les plus récentes ont été réalisées par l'INSD et la Banque Mondiale (2007) qui ont fait une analyse de la pauvreté par les avoirs et de la pauvreté subjective à l'échelle des individus, puis par Kaboré et al. (2009) qui ont analysé la pauvreté multidimensionnelle à l'échelle des ménages. L'implication de l'anthropologie est encore embryonnaire.

13 On peut donc estimer que si les politiques de développement économique et social mises en œuvre dans la lutte contre la pauvreté ont montré leurs limites, ce n'est pas seulement en raison de la complexité du phénomène de pauvreté jugé multidimensionnel, c'est aussi dû à la faiblesse de sources scientifiques autres qu'économiques, d'approches théoriques disponibles pouvant guider la prise de décision efficiente. La pauvreté ne se réduit pas uniquement à l'insuffisance des revenus. Elle ne peut se satisfaire de considération simplement économique. Les comportements et attitudes hérités de la culture sont des facteurs extrêmement importants. Quelle contribution l'anthropologie peut-elle alors apporter aux stratégies de développement de lutte contre la pauvreté?

\section{La nécessaire participation de l'anthropologie}

Il serait peut-être exagéré de lier l'échec des politiques successives de développement et de lutte contre la pauvreté (Programme d'ajustement structurel, Cadres Stratégique de lutte contre la pauvreté, etc.) entreprises au Burkina Faso à la seule absence de l'anthropologie dans leur mise en œuvre. Cependant, le constat est que la prise en compte des aspects qualitatifs est très souvent insuffisante ou extrapolée à partir des données quantitatives. Une intégration des sciences sociales notamment de l'anthropologie non seulement au niveau de la recherche mais aussi dans la mise en œuvre des politiques est donc nécessaire. Cette philosophie devrait permettre de : «Servir d'intermédiaires culturels (« courtiers») entre le monde du développement et celui de la communauté, recueillir les savoirs et les points de vue locaux, situer les communautés locales et les projets, dans des contextes plus larges d'économie politique, appréhender holistiquement la culture. Ce sont là autant de contributions importantes, sinon indispensables, de l'anthropologie au processus du développement »(Escobar, $1997: 544)$.

15 L'analyse anthropologique des déterminants actuels de la dynamique des sociétés permet d'envisager de façon prospective, des scénarios de changements sociaux. Certes la socio-anthropologie du développement et du changement social, qui s'associe à l'anthropologie d'intervention ou l'anthropologie appliquée est une approche relativement récente. " $\mathrm{La}$ socio-anthropologie du développement, fortement liée à l'Afrique, et que l'on peut aussi appeler « nouvelle anthropologie du développement ", a pris forme dans les années 1980 » (Olivier de Sardan, 2007 : 543). Elle utilise différentes méthodes propres à l'ethnologie classique pour mieux éclairer les attentes des populations et des acteurs de développement. II s'agit d'étudier "les interactions entre " développeurs " et " développés ", et les stratégies d'acteurs appartenant à des mondes sociaux très variés mis en relations par les pratiques et les politiques de développement " (Olivier de Sardan, 2007 : 547). C'est pourquoi, afin de mieux rendre compte des perceptions, l'implication de 
l'anthropologie pour être efficace, doit aller au-delà de l'approche dite participative utilisée dans la "recherche-action de salon » utilisée pour la mise en œuvre de décision politiques. L'anthropologue doit recueillir l'appréciation des populations sur leur propre condition de vie, analyser leur connaissance des moyens mis en œuvre par les pouvoirs publics à leur profit. Cela permet de déterminer des stratégies efficace a mettre en œuvre.

16 La réalité actuelle est que les perceptions des populations ne semblent pas être suffisamment prises en compte dans les politiques de lutte contre la pauvreté parce que la recherche qui s'occupe de ce volet n'est pas mise en avant. Les considérations économiques semblent primer sur celles sociales et culturelles.

17 Selon Rollet, Simmel, considéré comme fondateur de la sociologie de la pauvreté définit " pauvre » celui qui est désigné comme tel par la société et qui bénéficie, à ce titre, de l'assistance (Rollet, 2008). La pauvreté est donc conçue comme une construction sociale qui se développe à partir des relations entre différents acteurs. Les perceptions selon les groupes de population deviennent alors fondamentales de ce point de vue. Il faut alors chercher à comprendre comment les individus euxmêmes se définissent dans cet état. La représentation sociale et individuelle du phénomène de la pauvreté est donc importante. Comme nous le montrions au début de ce texte, dans les différentes sociétés du Burkina Faso l'analyse du corpus des appellations correspondant à la notion de pauvreté permet de constater que pour les uns, c'est une souffrance liée à une situation qui engendre des difficultés de vie, pour d'autres, c'est un handicap, une santé défectueuse ou un rang social défavorable au sein de la communauté. Par exemple de nos jours, le mot talga en mooré désigne une personne démunie sur le plan économique. Or, à l'origine, il servait à distinguer une personne ordinaire du noble. Ces exemples montrent la diversité des perceptions d'une situation de précarité.

18 L'implication de l'anthropologie dans l'approche du développement de la pauvreté permet donc à travers l'analyse des perceptions, d'aller au-delà de la vision purement matérielle et de prendre en compte les aspects culturels. Ces perceptions qui s'expriment à travers les discours quotidiens, les systèmes d'appellations et autres modes de désignations, permettent de voir que la pauvreté est appréhendée autant sous l'angle de la précarité sociale que du manque matériel. Lors d'un de nos séjours au village, un de nos oncles à qui nous demandions pourquoi il ne venait pas en ville, nous répondit a peu près ceci : «Ici je me sens bien. Si j'arrive à remplir mes deux greniers à l'issue d'une bonne saison des pluies, je suis heureux. Je vais au marché, je vais aux manifestations socioculturelles du village, et lorsque le temps est très chaud je discute et somnole sous l'arbre à palabre. Je n'ai besoin de rien sauf de temps en temps de quoi m'offrir une calebassée de bière de mil. Tant que je me porte bien et que je mange à ma fin je suis heureux. En ville il faut tout acheter et personne n'a le temps. Pour rien au monde je n'irais vivre là-bas. " Peut-on mesurer le bien être d'un tel individu en terme d'insuffisance de biens matériels ou d'absence d'un certain nombre de service sociaux de base dans son environnement? Cet individu qui est loin d'être considéré comme pauvre par ses proches et lui-même, ne saurait échapper à cette catégorie si on lui applique une unité de mesure économique pour évaluer sa pauvreté. Par exemple la famille nombreuse synonyme de richesse sociale du point de vue culturel peut dans certaines circonstances constituer un facteur aggravant 
de la pauvreté de l'individu si les personnes à charges sont dans l'incapacité de participer à la production. On retiendra de ce qui précède que la représentation socio-culturelle de la pauvreté est essentiellement dynamique et doit être comprise comme telle pour toute politique de transformation sociale.

19 Afin de répertorier les perceptions des individus et mieux maîtriser leur vécu, la méthode basée sur les histoires de vie ou récits de vie peuvent être bien indiquées car elle privilégie la description des conditions d'existence des populations d'étude dans une culture spécifique et à un moment donné de leur histoire. Cette pratique orale tente donc d'identifier les causes et les motifs variés qui se dégagent du discours qui affère un sens à la série d'évènements vécus par le sujet. Elles produisent des savoirs spécifiques en cherchant à impliquer les thèmes sur lesquels se déroule la recherche. Il s'agit d'explorer le processus de construction du sujet au sein de l'espace social : comment il donne un sens aux situations et aux événements de son existence, comment il agit et se construit dans son environnement historique, social, culturel, politique. La recherche biographique ne vise pas tant à produire un savoir objectivé qu'à comprendre la manière dont les acteurs font pour signifier leurs expériences dans le domaine d'observation, dans le cas de la pauvreté et le rôle que jouent les acteurs externes tel que l'Etat. L'objectif final de l'enquête est d'identifier les éléments influant sur les modalités objectives et ressenties du maintien dans la pauvreté, des choix exogènes subits par l'individu et son groupe, des stratégies passées envisagées. Alliée à l'observation participante qui permet une maîtrise du milieu, cette méthode permet d'aller au-delà des stratégies individuelles et d'apprendre la dynamique du milieu à travers des exemples de cas.

20 La confrontation des histoires vécues par un certain nombre d'individus dans le même environnement socio-culturel permet de tirer des occurrences dont l'analyse peut fournir des informations pertinentes sur la voie à suivre, accompagner la dynamique sociale dans le sens d'un changement positif.

21 La longueur d'une telle procédure de recherche expliquerait sa non prise en compte dans les recherches d'information en prélude à la mise en œuvre des politiques de développement. Au Burkina Faso, dans le cadre du Cadre Stratégique de lutte contre la pauvreté (CSLP), des fora ont été organisés au niveau régional afin d'amender un document qui a servi de base au document national. Des assises nationales ont été organisées par la suite dans la capitale où plus d'un milliers de personnes de niveaux scolaires disparates et d'origines socio-professionnelles diverses ont été invités à amender et adopter ce document national qui a servi de base à la mise en œuvre de la stratégie nationale de lutte contre la pauvreté. Cette "méthode participative » qui s'est déroulée pendant trois jours dans des salles de conférence climatisées ne pouvait que conduire à des resultats mitigés du CSLP. Ce qui a nécessité la mise en œuvre d'un nouveau programme dénommé : Stratégie de croissance accélérée et de développement durable. Lorsque les bases de collecte des informations sont "faussées" parce que n'intégrant pas les éléments d'une recherche conséquente sur les perceptions et les attentes des populations, les stratégies adoptées ne peuvent qu'être difficilement en adéquation avec les attentes de ces populations. En voulant gagner du temps on prend des raccourcis qui contournent les problèmes véritables des populations. Alors on fait des investissements coûteux qui n'ont pas toujours des effets probants sur 
l'amélioration de leurs conditions de vie, toutes choses qu'une plus grande implication de l'anthropologie et des disciplines voisines aurait permis d'éviter à bon escient.

\section{Conclusion}

Il n'est pas exagéré de dire que si la recherche scientifique a été présente au Burkina Faso dans l'accompagnement des programmes de développement et de lutte contre la pauvreté, cela ne s'est fait que dans une optique quantitative. Cinquante ans après son accession à l'indépendance, le Burkina a mis en œuvre de nombreuses stratégies socio-economiques qui visent à apporter un mieux être aux populations. Celles-ci ont connu des fortunes diverses à causes de la non prise en compte des perceptions et attentes des populations. Du reste, ces recherches qui n'ont pas eu un caractère national sont demeurées dans le domaine de ce qui est couramment appelé «littérature grise» contrairement aux recherches économiques et statistiques qui ont souvent donné lieu à des publications scientifiques. Les recherches en socio-anthropologie sont souvent jugées à tort ou à raison trop longues à mettre en oeuvre et aboutissant à des résultats difficilement applicables à une échelle nationale. Cependant, avec les échecs répétés des politiques de développement et de lutte contre la pauvreté, l'Etat et ses partenaires financiers sont de plus en plus attentifs aux «volets qualitatifs » des études qui précèdent et/ou accompagnent les actions de développement. Il importe donc aux chercheurs en socio-anthropologie de monter leur capacité à mener des recherches et des réflexions à l'échelle nationale sur les problèmes clés de développement. En intégrant des innovations méthodologiques et conceptuelles adéquates avec rigueur et perspicacité, ils répondront aux attentes des décideurs et partenaires au développement tant en contribuant au renforcement scientifique de leur discipline.

\section{BIBLIOGRAPHIE}

Baulch B. and Hoddinott, J. 2000. « Economic Mobility and Poverty Dynamics in Developing Countries ", Journal of Development Studies, 36 (2): 1-24.

Chambers, R \& A. Pacey, L.A. Thrupp 1994. Les paysans d'abord : les innovations des agriculteurs et la recherches agronomiques, Paris, Karthala.

Chauveau J.-P., Cormier-Salem M.C., Mollar, E., 1999. L'innovation en agriculture. Question de méthodes et terrains d'observation, Paris, IRD.

Corcoran M., Ducan G.J., Curin G., Curin P., 1985. « Myth and Reality : the causes and persistence of Poverty ». Journal of Policy Analysis and Management, 4 (4) : 516-536.

Crush J., 1995. « Introduction: Imagining development. », in Crush J. (ed.), Power of development. New York, Routledge :1-26. 
Datt G., Ravallion M., 1992. « Growth and Redistribution Components of Changes in Poverty Measures: decomposition with applications to Brazil and India in 1980s ", Journal of Development Economics, 38 (1992) 275-295.

De Janvry A. and Sadoulet E., sept. 2000. « Growth, Inequality and Poverty in Latin America : a causal analysis ", 1970-1994. Review of Income and Wealth, 46 (3).

Denis H. 1951. La monnaie, les lois fondamentales du capitalisme, Paris, ed. sociales, Coll. la Culture et les Hommes.

Dia M., 1991. « Développement et valeurs culturelles en Afrique subsaharienne », Finance et Développement, Décembre, 10-13.

Diakité S., 1985. Violence technologique et développement, Paris, L'Harmattan.

Diawara, F., 1972. Le manifeste de l'homme primitif, Paris : Grasset, Montréal : Lemeac.

Diop C. A., 1986. « Analyse critique des critères et ou paramètres retenus pour définir les aires culturelles africaines ", in Spécificités et convergences culturelles dans l'Afrique au Sud du Sahara, Paris, Unesco, 43-55.

Dockès P. et Rosier B. 1988, «La question du développement face à l'histoire » dans Développement endogène : aspects qualitatifs et facteurs stratégiques, Paris, UNESCO, 87-108.

Dorothée Buccanfuso, Tambi Samuel Kaboré, 2004. «Croissance, Inégalité et Pauvreté dans les années 90 au Burkina Faso et au Sénégal ». Revue d’Économie du Développement, Vol 2, pp 9-36, juin 2004.

Escobar A., 1997. « Anthropologie et développement », Revue Internationale des sciences sociales, $n^{\circ} 154:$ 539-556.

Grimm M., Günther I., 2006. «Growth and Poverty in Burkina Faso: a Reassessment of the paradox. ", Journal of African Economies, 16 (1): 70-101.

INSD et la Banque Mondiale, 2007. Dynamique de la pauvreté par les avoirs des ménages au Burkina Faso : une analyse à partir des enquêtes auprès des ménages 2003-2007. MEF, INSD, BM, Septembre 2007.

Jalan, J., Ravallion, M., 2000. Determinants of Transient and Chronic Poverty: Evidence from Rural China. Development Research Group, World Bank, Washington, DC.

Jayaraman R., Lanjouw P., Feb. 1999. The evolution of Poverty and Inequality in Indian Villages. The World Bank Research Observer, 14 (1): 1-30.

Lachaud J. P., 2002a. « Pauvreté monétaire et privations de capacités au Burkina Faso » Série de recherche $\mathrm{n}^{\circ} 6$, Centre d'économie du développement. Université Montesquieu - Bordeaux IV.

-. 2002b. « Dépenses des ménages, développement humain et pauvreté au Burkina Faso : substitution ou complémentarité ? » Centre d'économie du développement. Université Montesquieu - Bordeaux IV.

Olivier de Sardan J.-P., 1995. Anthropologie et développement. Essai en Socio-Anthropologie du Changement, Paris, Khartala.

-. 2007. «De la nouvelle anthropologie du développement à la socio-anthropologie des espaces publics africains ", Revue Tiers Monde, $\mathrm{n}^{\circ} 191$ : 543-552.

Rentel, U; Langeheine, R and Bernsten, R. 1998. The Estimation of Poverty Dynamics Using Different Measurements of Household Income. Review of Income and Health 44(1), March 1998. 
Sawadogo K., 1997. La pauvreté au Burkina Faso : une analyse critique des politiques et des stratégies d'intervention locales. ECDPM Document de travail $n^{\circ} 51$.

Sawadogo K., J.-B. Ouédraogo et T. Thiombiano, 1995. Profil de la pauvreté au Burkina Faso : Une approche qualitative et quantitative. Rapport à la Banque Mondiale. Université de Ouagadougou. Juillet 2005.

Stevens A.H., 1999. Climbing out of poverty, Falling Back in: Measuring the persistence of poverty over multiple spells. The journal of human resources, 34 (3) : 557-588.

-. The dynamics of poverty spells : Updating Bane and Ellwood. The American Economic Review, 84

(2): 34-37.

Using Different Measurements of Household Income. Review of Income and Wealth, 44 (1), pp. 81-98.

Wetta, C., Kaboré T.S., Bonzi K.B., Sikirou S., Sawadogo M., Somda P., 1999. Profil d'inégalité et de pauvreté au Burkina Faso. Cahiers de Recherche du CREFA, N $: 00-02$, Jel : D33, I32, Université Laval, Québec.

\section{NOTES}

1. Estimations effectuées à l'aide du modèle de simulations PAMS, MEF/DGE, IAP mars 2009.

2. "l'une des impulsions fondamentales de ceux qui écrivent sur le développement, (en tant que domaine d'étude et en tant que pratique), est le désir de définir, de catégoriser et de mettre de l'ordre dans un champ de significations hétérogènes qui ne cesse de s'étendre. " (Crush, 1995: 2).

\section{RÉSUMÉS}

$\mathrm{Au}$ Burkina Faso, les études nationales sur le développement et la lutte contre la pauvreté sont le plus souvent effectuées par des économistes. La mesure de la pauvreté est alors une question de statistiques et de données économétriques quantitatives. Les quelques rares études scientifiques qualitatives qui ont pu être entreprises depuis les années 90 sont restées souvent assez parcellaires et ne concernent que quelques deux à trois régions. Toutefois, elles ont montré l'importance de la prise en compte des perceptions des populations, voire de leur culture dans la compréhension du phénomène. A cet effet, concernant la recherche nationale sur la pauvreté, l'intervention de l'anthropologie doit permettre d'articuler les dimensions sociales, politiques et spatiales et d'inscrire la pauvreté dans le contexte global des problèmes de la société.

In Burkina Faso, national studies on development and poverty are most often conducted by economists. The measurement of poverty is hence a question of statistics and quantitative econometric data. The few qualitative studies carried out since the 1990s remain partial as they concern only two or three regions. However, they have demonstrated the importance of taking into account people's perceptions of the phenomenon. The intervention of anthropology in national poverty research would allow for the articulation of social, political and spatial dimensions and inscribe poverty in the global context of societal problems. 